\title{
P214 IMPROVEMENT OIL RECOVERY FROM RESERVOIRS BY GASEOUS AGENT INJECTION
}

A. GRITSENKO, R. TER-SARKISOV, A. SHANDRYGIN and O. VLASENKO Ail-Russian Scientific Research Institute of Natural Gas and Gas Technology Leninsky District, p. Razvilka, 142717 Moscow Region, Russia

The gas agents injection into oil deposits is one of the effective methods of the development. The oil recovery from reservoir at gas injection occurs under conditions of the multicontact miscible oil displacement. In general it can be marked out two main processes of miscible displacement: with prevalence of: 1) hydrocarbons evaporation out of oil and 2) condensation of injected components gas into oil. In the first case the oil recovery from formation comes true at the expense of gas drive and partially at the expense of evaporation intermediate hydrocarbon components into gas; in other case- the liquid phase "bank" (100\% liquid saturation ) is formed in formation and oil is displaced like piston.

Problem of efficiency oil displacement by gaseous agents for fractured-porous media is especially important. These reservoirs consist of fractures and matrixes and their permeability and porosity are able distinguished a few orders. Therefore at the gas injection into fracturedporous media fast gas breakthrough onto high-permeability fractures with small coverage of displacement from low-permeability blocks are possible. We executed research of process of miscible oil displacement by gas from fractured-porous reservoirs under various conditions; criteria of technological efficiency of this process are established and technological decisions to optimise the gaseous agents injection in such formation are also developed.

The research was carried out on the basis of numerical simulation of twophase 
multicomponent of hydrocarbons flow in double porous medium. We used the differential material-balance equation for Component $k$ in Medium $i$ :

$$
\begin{aligned}
& \nabla\left(\lambda_{g} C_{k g} \nabla P_{g}+\lambda_{L} C_{k L} \nabla P_{L}\right)_{i}=\frac{\partial}{\partial t}\left(\alpha m \rho C_{k}\right)_{i}+\left(Q_{e x}+Q\right)_{k}(2-i) \\
& \text { where: } \quad \lambda_{\beta}=\frac{k k_{\alpha} \rho_{\alpha}}{\mu_{\alpha}} \quad \rho=\rho_{g}(1-S)+\rho_{L} S=F\left(P, C_{1} C_{2} \ldots C_{N}\right) \\
& Q_{e x_{k}}=\left(q_{g} \rho_{g} C_{k g}+q_{L} \rho_{L} C_{k L}\right)_{e x} \quad Q_{k}=q_{g} \rho_{g} C_{k g}+q_{L} \rho_{L} C_{k L} \\
& \beta \text { - phase }(\beta=g \text { - gas, } \beta=L \text { - liquid }) ; i \text { - media }(i=1 \text { - fracture, } i=2 \text { - matrix }), \lambda-
\end{aligned}
$$
mobility parameters; $k_{,} k_{\alpha}$ - permeability and relative permeability; $\rho$-density; $c$-mass or mole concentration (fraction) of components; $\alpha$ - fraction of media (fracture, porous matrix); $\mu$ - viscosity; $m$ - porosity; $q_{e x}$ - exchange rate between fracture and matrix ; $q$ - production or injection rate; $N$ - number of components; $P$ - pressure; $t$ - time; $S$ - liquid saturation; $q_{e x}=\omega\left(p_{2}-\right.$ $\left.p_{1}\right), \quad \omega$ - factor of crossflow fluids exchange intensity between media of a reservoir.

Separate phase equilibrium relationships is applied for the matrix and fractured medium:

$$
\begin{aligned}
\sum_{1}^{N} C_{k} & =1 \quad \sum_{1}^{N} x_{k}=1 \quad \sum_{1}^{N} y_{k}=1 \\
x_{k_{i}} & =K_{k}^{g l} y_{k_{i}}, K \text { - constant of phase equilibrium. }
\end{aligned}
$$

The differential material-balance equations solutions were performed by using finitedifference method according to the scheme of implicit pressure and explicit concentrations. To determine the phase equilibrium and fluid-property in equation the Peng-Robinson equation of state was used. The special procedure to accelerate computations was employed.

The investigations were executed for a case of a $1 \mathrm{D}$ flow of variants with different reservoir pressure and temperatures, rates of the gas injection. We assigned the oil mix model consisting of three components: $C_{1}, C_{3}$ (simulates components $C_{2-4}$ ) and pseudo-component $\mathrm{C}_{5+}$. Boiling point of a pseudo-component $-\mathrm{C}_{5}+$ made $386 \mathrm{~K}$, density $-700 \mathrm{~kg} / \mathrm{m}^{3}$, molecular weight -106 . In calculation variants different shares of these components in initial oil structure 
were posed: dry hydrocarbon gas, enriched hydrocarbon gas and carbon dioxide as the gaseous agents were considered. The dry gas injected under pressure approached to bubblepoint, and enriched hydrocarbon gas and carbon dioxide under $0,6-0,7$ of bubble-point saturation pressure. The permeability of fractures to matrixes ratio $\left(k_{1} / k_{2}\right)$ is varied from 10 to 10000 , length of the block (L) from 1 to $20 \mathrm{~m}$. To analyse the data parameters $\alpha_{1}$ and $\alpha_{2}$ was used also, representing a share of fractures and matrixes in a formation and connected with blocks and fractures size. Relative phase permeabilities and the capillary pressure were set as:

$$
\begin{aligned}
& k_{L}=\left(\left(S_{L}-S_{L 0}\right) /\left(1-S_{L 0}\right)\right)^{\text {al. }} \quad \text { at } S_{L}>S_{L 0}, \quad k_{L}=0 \quad \text { at } S_{L}<S_{L 0} \\
& k_{\mathrm{g}}=\left(\left(\mathrm{S}_{\mathrm{g}}-\mathrm{S}_{\mathrm{g} 0}\right) /\left(1-\mathrm{S}_{\mathrm{g} 0}\right)\right)^{\mathrm{ag}} \quad \text { at } \mathrm{S}_{\mathrm{g}}>\mathrm{S}_{\mathrm{g} 0}, \quad \mathrm{~K}_{\mathrm{g}}=0 \quad \text { at } \mathrm{S}_{\mathrm{g}}<\mathrm{S}_{\mathrm{g} 0} \\
& \mathrm{p}_{\mathrm{k}}=\mathrm{p}_{\mathrm{ko}} \mathrm{s}^{\mathrm{akk}} \quad \text { at } \mathrm{S}_{\mathrm{L}}>\mathrm{S}_{\mathrm{L} 0}, \mathrm{p}_{\mathrm{K}}=\mathrm{p}_{\mathrm{ko}} \mathrm{S}_{\mathrm{Lo}}{ }^{-2 \mathrm{k}} \quad \text { at } \mathrm{S}_{\mathrm{L}}<\mathrm{S}_{\mathrm{LO}}
\end{aligned}
$$

For fractures $-\mathrm{S}_{\mathrm{L} 0}=0.15, \mathrm{~S}_{\mathrm{g}} \mathrm{O}_{\mathrm{g}}=0.1, \mathrm{a}_{\mathrm{L}}=1.25, \mathrm{a}_{\mathrm{g}}=1.25, \mathrm{p}_{\mathrm{ko}}=200 \mathrm{~Pa}, \mathrm{a}_{\mathrm{k}}=5,0 . \mathrm{S}_{\mathrm{L} 0}{ }^{0.85}$, and for matrixes- $\mathrm{S}_{\mathrm{LO}}=0.35, \mathrm{~S}_{\mathrm{g}} 0_{\mathrm{g}}=0.25, \mathrm{a}_{\mathrm{L}}=2.5, \mathrm{a}_{\mathrm{g}}=2.5, \mathrm{p}_{\mathrm{ko}}=1000 \mathrm{~Pa}, \mathrm{a}_{\mathrm{K}}=10,0 . \mathrm{S}_{\mathrm{L} \theta}^{0.75}$ were accepted.

The research allowed to establish the following main laws of multicontact miscible oil displacement by gas from fractured-porous reservoirs. There are main parameters determining efficiency of oil drive by gas from fractured-porous reservoirs: ratio of permeabilities, porosity, share both of fractures and matrixes of a formation.

At miscible displacement with prevalence of evaporation one more factor determining process of displacement is the evaporating into gas intermediate components content in oil. The fronts of oil displacement by gas advance in fractures and blocks is supervised by a ratio of permeabilities and porosities of media and shares of media in a formation also. The connection between a ratio of rates of fronts distribution of oil displacement in media of a formation and parameters emerges $\sigma=\alpha_{1} m_{2} k_{1} /\left(\alpha_{2} m_{1} k_{2}\right)$ and $\theta=m_{2} k_{1} /\left(m_{1} k_{2}\right)$. It is confirmed by data on liquid saturation distribution submitted on fig. 1 for several variants of calculations with an oil mix of the following initial structure (\% mole): $C_{1}-45,0, C_{3}-25,0, C_{5+}-30,0$. The quantity recovered oil from a formation is defined in volumes of the agent injection through fractures and blocks. Hence the oil recovery depends on the parameter $\sigma$ and gas 
evaporating ability. On fig. 2 dependence upon parameter $\tau$ from $\sigma$ is shown for various shares of intermediate components $\mathrm{C}_{24}$ (in this case $\mathrm{C}_{3}$ ) in oil. Parameter $\tau$ represents the maximum value of relation of oil volumes extracted from a formation and injected agent (both values are reducted to reservoir conditions). Parameter $\sigma$ expresses a filtration ratio and capacitor properties of media of a reservoir. The value reduction of parameter $\tau$ with increase $\sigma$ is coursed of injection more significant volumes of gas through a formation fracture with small matrixes displacement coverage. The increase of parameter $\tau$ with increase of a share of components $\mathrm{C}_{2-4}$ occurs at the expense of additional volume of oil recovery with gas because of evaporation intermediate components.

The injection of the working agent under pressure above minimum pressure of multicontact mixture (MMC) under given temperature is the necessary condition of effective miscible displacement with prevalence of components condensation out of gas. The formation in fractures and blocks of a zone with $100 \%$ liquid saturation is supervised by parameter $\theta$. Therefore the full mixture in blocks can be attained over considerably smaller plots of a formation compare to fractures (fig. 3 - injection carbon dioxide case). The value of oil volumes recovered out of a formation ratio to injected gas ( $\tau$ ) depends on parameter $\sigma$ and liquid saturation $\left(\mathrm{S}_{\mathbf{L}}\right)$ to start an gaseous agent injection. Dependence on parameter $\tau$ from value $\sigma$ and $\mathrm{S}_{\mathrm{L}}$ is submitted on fig. 4 .

The additional oil output from fractured-porous media at miscible displacement with prevalence of evaporation can be achieved at the expense of the gas injection in a cyclic mode. The mass transfer between fractures and matrixes in a formation is intensified in this case at the expense of periodic change of size and fluids flows direction. 


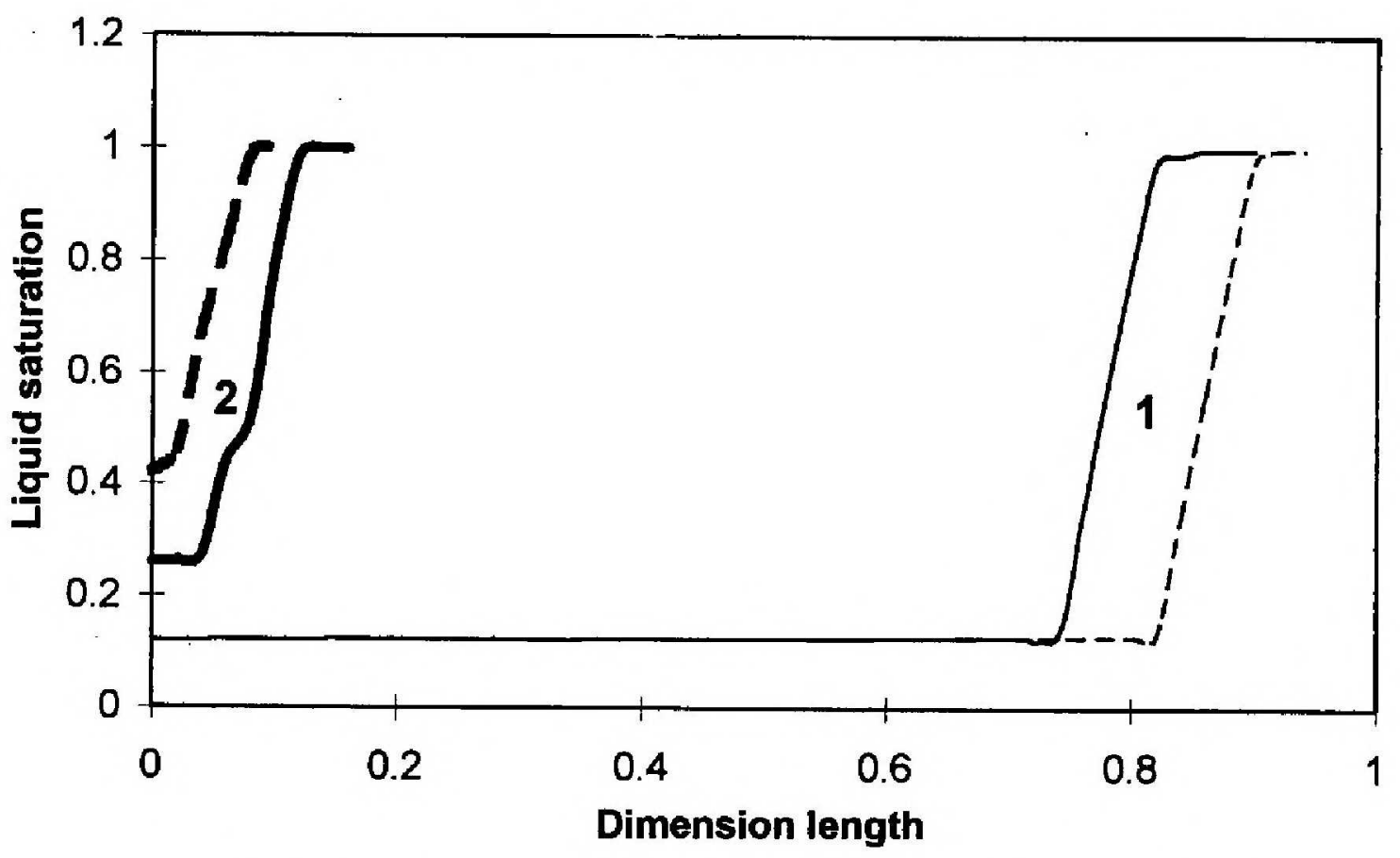

Fig.1 Distribution of liqid saturation in fractured (1) and matrix (2) for $\sigma=10^{-3}, Q=1000$ (dashed line) and $\sigma=10^{-4}, Q=100$ (solid line).

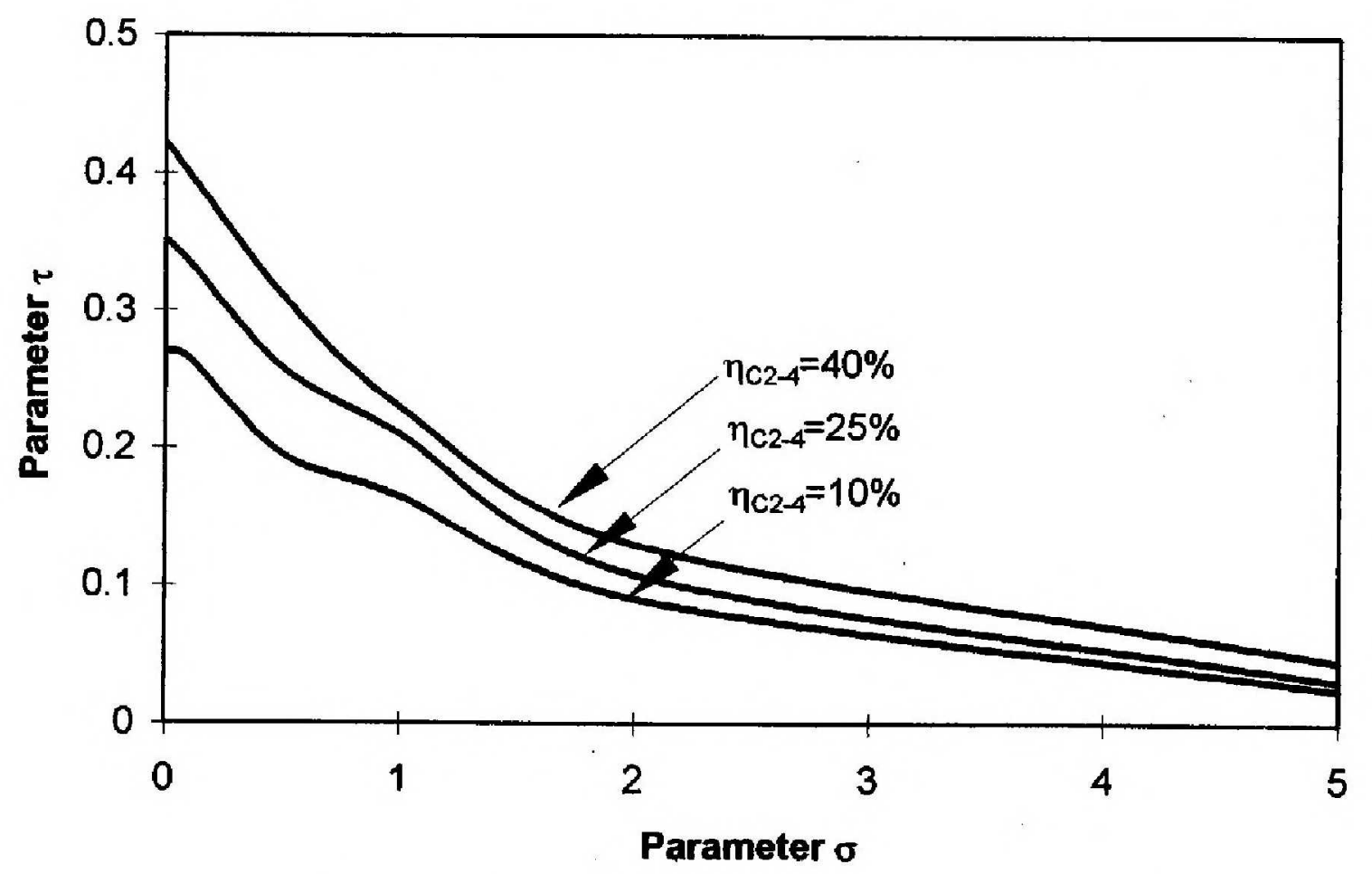

Fig.2 Parameter $\tau$ vs. parameter $\sigma$ by various concentration $\eta \mathrm{C}_{2-4}$. 


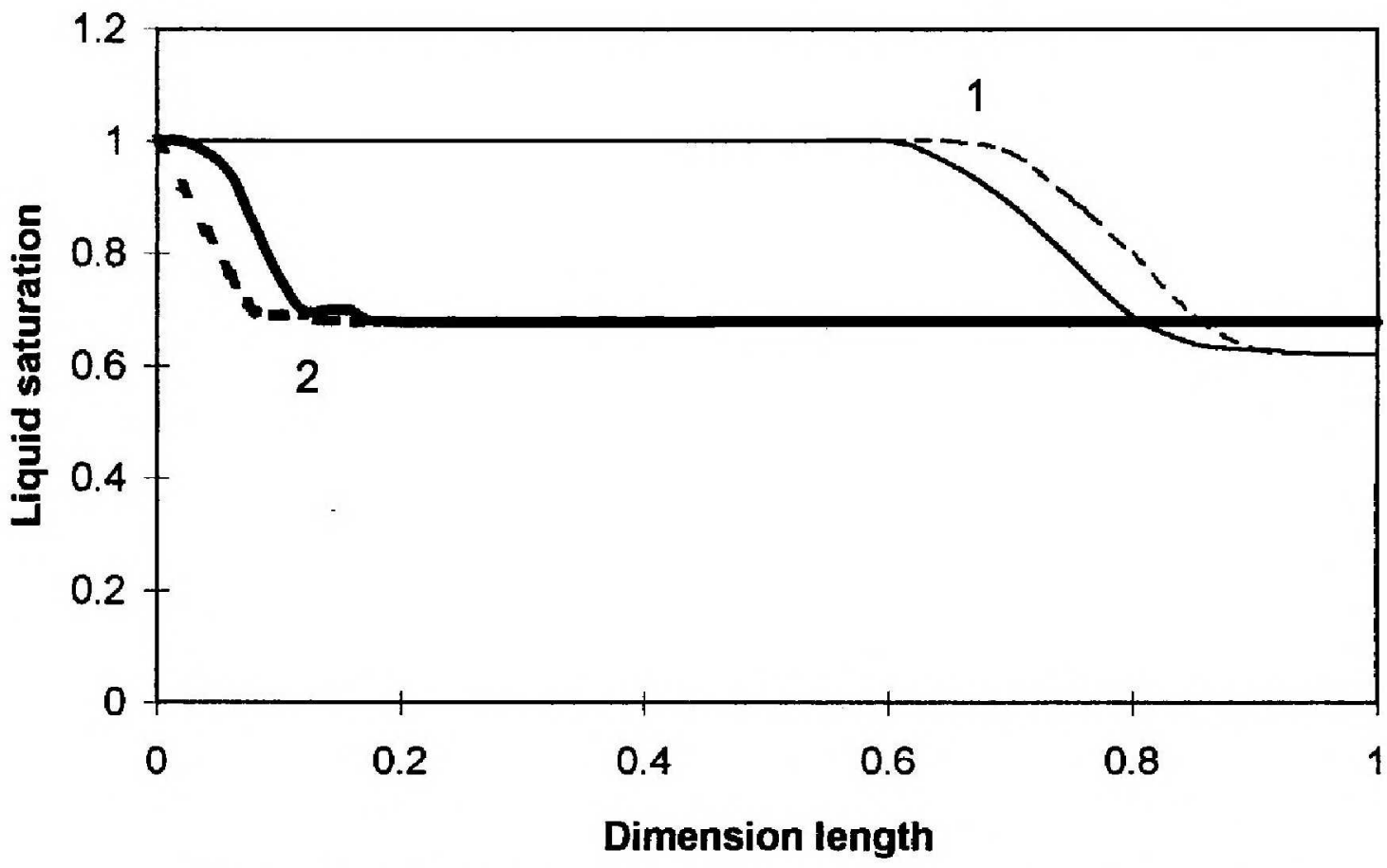

Fig.3 Distribution of liquid saturation in fractured (1) and matrix (2) for $\sigma=10^{-3}, Q=1000$ (dashed line) and $\sigma=10^{-4}, Q=100$ (solid line).

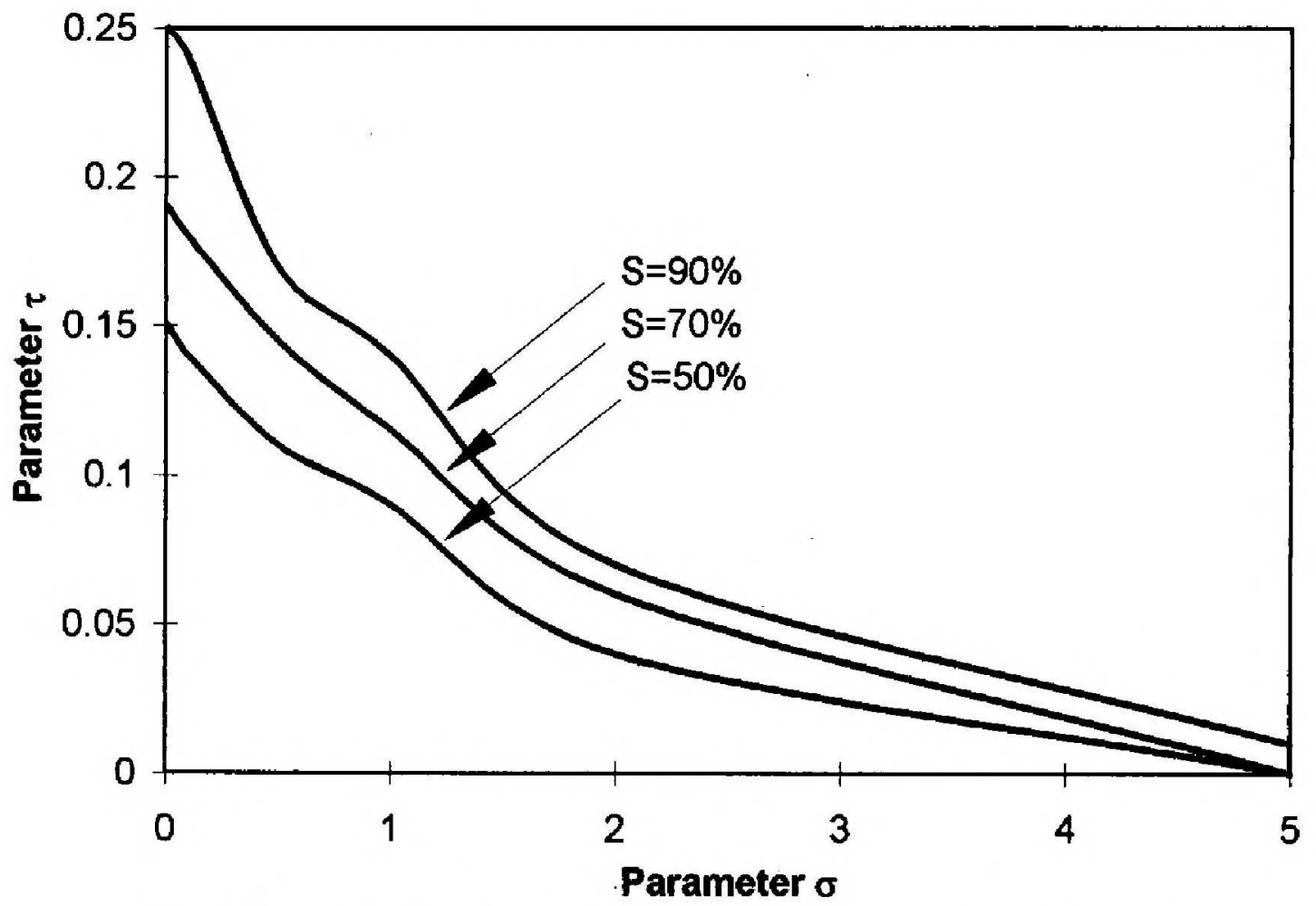

Fig.4 Parameter $\tau$ vs. parameter $\sigma$ by various liquid saturation $\mathbf{S}$. 